\title{
Sleep quality and body weight status of Malaysian university students
}

\author{
Nurul Fareeza Suhaimi', Zuriati Ibrahim ${ }^{1 *}$, Siti Nur 'Asyura Adznam ${ }^{1}$ \& \\ Sabariah Md Noor ${ }^{2}$
}

${ }^{1}$ Department of Dietetics, Faculty of Medicine and Health Sciences, Universiti Putra Malaysia, 43400 Serdang, Selangor Malaysia; ${ }^{2}$ Department of Pathology, Faculty of Medicine and Health Sciences, Universiti Putra Malaysia, 43400 Serdang, Selangor Malaysia

\begin{abstract}
Introduction: Inadequate sleep duration is a modifiable behaviour linked with body weight, yet limited is known on the role of sleep quality with body weight status among university students. The study aimed to determine sleep quality and its association with body weight status among university students. Methods: A crosssectional study was conducted among 240 university students $(24.6 \%$ males, $75.4 \%$ females) with a mean age of $21.22 \pm 1.24$ years. Subjects were recruited from four faculties of University Putra Malaysia (UPM). Information on socio-demographic, sleep quality and anthropometric measurements were collected. Pittsburgh Sleep Quality Index (PSQI) was used to assess sleep quality, whereby a summation score from seven components yielded a global score on sleep quality, with higher scores indicating poor sleep quality. Results: Majority of subjects were Malay $(79.2 \%)$ with a mean body mass index of $22.6 \mathrm{~kg} / \mathrm{m}^{2}$. A majority $(61.3 \%)$ had normal body weight, with the prevalences of underweight (UW), overweight (OW), and obesity (OB) at $12.9 \%, 19.6 \%$ and $6.3 \%$, respectively. The mean global PSQI score was $5.76 \pm 2.64$, with half of the respondents $(50.4 \%)$ experiencing poor sleep quality. OW-OB experienced poorer sleep quality $(6.53 \pm 2.79, p=0.004)$ and longer sleep latency $(1.34 \pm 0.10, p=0.008)$ relative to UW-NW. OB subjects had substantially more frequent sleep disturbances compared to non-OB subjects $(1.53 \pm 0.64, p=0.012)$. Conclusion: Findings suggest that OW-OB students at university are at a greater risk of having reduced sleep quality with longer sleep latency and frequent sleep disturbances. There is a need for a wellness initiative to reduce the incidence of obesity while fostering healthier sleeping habits among university students.
\end{abstract}

Keywords: Sleep quality, university student, body weight status

\section{INTRODUCTION}

Malaysia is leading in the prevalence of adult obesity compared to other Southeast Asian countries, with nearly half of its adult population having a body mass index (BMI) of above $25 \mathrm{~kg} / \mathrm{m}^{2}$ (IPH, 2019). Several initiatives have been made by the government to tackle the obesity issue such as the implementation of My body fit and fabulous MyBFF@ home and MyBFF@school (Mohamad Nor, Ambak \& Aris, 2018) to encourage healthy eating habits and active lifestyle. However, the root factors for obesity

\footnotetext{
*Corresponding author: Dr Zuriati Ibrahim

Department of Dietetics, Faculty of Medicine and Health Sciences, Universiti Putra Malaysia, 43400 Serdang, Selangor, Malaysia Tel: (6)03-97692464; Fax: (6)(03)-89426769; E-mail: zuriatiib@upm.edu.my doi: https: / / doi.org/10.31246/mjn-2020-0048
} 
among the Malaysian population may not have been properly addressed as the prevalences of overweight and obesity (OW-OB) have consistently increased (IPH, 2015). This is observed in a series of National Health and Morbidity Survey (NHMS) conducted among the Malaysian population aged $\geq 18$ years old, which showed consistent elevation trends in the prevalences of OW-OB from $29.1 \%$ and $14.5 \%$ in 2006 (IPH, 2008) to 30.0\% and $17.7 \%$ in 2015 (IPH, 2015), and more recently to $30.4 \%$ and $19.7 \%$ (IPH, 2019), respectively.

Previous studies have suggested that the trend of obesity may start from as early as two years old (Robinson et al., 2019), which then develops in a consistent trajectory pattern when entering university life (Vadeboncoeur, Townsend \& Foster, 2015). Poor sleep quality has been associated with poor academic performance (Azad et al., 2015), impaired judgement (Greer, Goldstein \& Walker, 2013), and to some extent OWOB (Krističevič, Štefan \& Sporiš, 2018; Peltzer \& Pengpid, 2017; Fatima, Doi \& Mamun, 2016). Based on observational studies, a consistent pattern has been observed in the association between sleep quality with OW-OB. In a meta-analysis aimed to examine the association between poor sleep quality with OW-OB, higher odds of being OWOB $(\mathrm{OR}=1.46,95 \% \mathrm{CI}: 1.24$ to 1.72$)$ were observed among respondents with poor sleep quality (Fatima et al., 2016). This is in agreement with a study involving 2100 undergraduates that showed poor sleep quality was associated with a higher likelihood of being OW-OB (OR $=1.45 ; 95 \% \mathrm{CI}: 1.14$ to 1.83 ) (Krističevič et al., 2018).

In Malaysia, several studies have found a high prevalence of poor sleep quality among Malaysian university students (Nurismadiana \& Lee, 2018; Tien Ngu et al., 2017). However, the association between poor sleep quality with body weight remains inconclusive. While several studies conducted among Malaysian university students in Malaysia failed to find significant associations (Kumar, Othman \& Jeppu, 2020; Lai \& Say, 2013; Ganesh Kamath et al., 2014), other local studies have noted the potential role of sleep quality in influencing body weight, but the association remains unclear (Kumar et al., 2020; Lai \& Say, 2013; Ganesh Kamath et al., 2014). For example, a study conducted among Malaysian private university students $(n=201$, mean age $21.55 \pm 2.13$ years old) noted a negative correlation between sleep quality score and body weight, suggesting that increasing body weight reduces overall sleep quality (Kumar et al., 2020). Though the finding was not statistically significant, the study suggested that higher body weight elevates the risk of obstructive sleep apnea that consequently worsens sleep quality (Kumar et al., 2020). Another local study conducted among Malaysian university students $(n=191$, aged between 18 to 25 years old) found that obese respondents had a higher tendency to overeat when they had poor sleep, suggesting a potential role for sleep in influencing appetite and eventually body weight (Greer, Goldstein \& Walker, 2013).

University phase is a critical period when university students establish modifiable health-related behaviours that persist into adulthood. Tien Ngu et al. (2017) demonstrated that students are especially vulnerable to poor sleep quality resulting from increased academic demands, extensive use of electronic devices, and stress (Azad et al., 2015). Therefore, this study was conducted to determine the association between sleep quality and body weight status among university students of Universiti Putra Malaysia (UPM). 


\section{MATERIALS AND METHODS}

A cross-sectional study was conducted among undergraduate students from four randomly selected faculties of UPM. A total of 240 eligible respondents were recruited based on a single proportion formula after considering $10 \%$ of missing data. The selected faculties consisted of three different fields of studies, namely sciences, arts, and technical background, guided by the Ministry of Higher Education's categorisation. A random generator software picked a total of four faculties out of 16. Two faculties were chosen for the sciences field because of its large proportion, followed by one faculty each for the arts and the technical fields. Institutional permissions were obtained, and updated name lists and contact information were sourced from the administration office. The study included participants if they were Malaysian undergraduates aged 18 to 25 years old. Those who were pregnant, lactating, or diagnosed with chronic diseases were excluded. Information sheet, agreement of confidentiality and bilingual questionnaires (English and Malay) were distributed to eligible respondents during data collection. Ethical approval was granted by the Ethics Committee for Research Involving Human Subjects, UPM (JKEUPM) [Ref: FPSK(EXP16)P169].

\section{Instruments}

This study involved collecting anthropometric data and utilised a selfadministered questionnaire to collect data on socio-demographic characteristics and sleep quality. Socio-demographic characteristics documented included age, gender, ethnicity, marital status, current residential, current year of study, the field of study, and faculty.

\section{Sleep quality}

Sleep quality was assessed using the Pittsburgh Sleep Quality Index (PSQI). PSQI consisted of seven component scores; 1) subjective sleep quality, 2) sleep latency, 3) sleep duration, 4) habitual sleep efficiency, 5) sleep disturbances, 6) use of sleep medication, and 7) daytime dysfunction over the past one month. Each component had a range of 0 to 3 points on a Likert scale, with " 0 " indicating no difficulty, while a score of " 3 " indicating severe difficulty. The seven component scores were then added to yield one "global" score, where a score of $\geq 5$ indicated "poor" sleeper. The Malay version of PSQI has been previously validated with a Cronbach's $\alpha$ of between 0.64 to 0.82 , indicating good internal consistency, and a testretest reliability of between 0.45 to 0.70 , indicating low to moderate reliability (Musa, Moy \& Wong, 2018; Yunus et al., 2017).

\section{Anthropometry}

Anthropometric measurements were performed by a qualified and trained researcher to avoid inter-researcher variability. Weight was measured using a digital weighing scale (OMRON model HBF-375) placed on a hard and balanced surface. During weight assessment, respondents were in light clothing, reminded to remove any footwear, and ensured that their pockets were emptied. Zero-calibration of the scale was ensured before measurement. Respondents were asked to stand in the centre of the scale without any support and weight was distributed evenly on both feet.

Height was measured using the SECA 214 mobile stadiometer with a measuring range of $20-207 \mathrm{~cm}$ (SECA 214). A stretch stature method was used 
whereby the respondent stood with feet and heels closed together, and ensured that buttocks and upper part of the back touched the scale.

The height and weight measurements were indexed into BMI using the equation of weight $(\mathrm{kg}) /$ height $\left(\mathrm{m}^{2}\right)$. BMI was classified according to the World Health Organization categorisation of body weight status (WHO, 2000).

\section{Statistical analysis}

IBM SPSS 22.0 was used for data analysis. Descriptive data for categorical variables were presented as frequency and percentage, while continuous variables were presented as mean and standard deviation. Apart from being guided by the central limit theorem, normality of assumption was considered met by visual inspection of the histogram or with the results of normality from Kolmogorov-Smirnov or Shapiro-Wilk tests. Mann-Whitney U test was used to determine the significant differences between two groups if the variable was not normally distributed, while KruskalWallis test was used to compare the differences between more than two variables. The level of significance was set at $p<0.05$.

\section{RESULTS}

\section{Socio-demographic characteristics}

A total of 240 respondents participated in the study. Table 1 shows the distribution of respondents according to their socio-demographic characteristics. The study population consisted of $24.6 \%$ males and $75.4 \%$ females, with a mean age of $21.22 \pm 1.23$ years. Majority of the respondents were Malay (79.2\%) and resided in the hostel, with an approximately equal distribution of students in their first (34.6\%), second $(31.7 \%)$ and third $(33.8 \%)$ years of study. In terms of body weight status, $12.9 \%$ were underweight (UW), 19.6\% OW, and
$6.3 \%$ obese. Majority of the participants $(61.3 \%)$ were of normal body weight.

Table 1. Socio-demographic characteristics of respondents, $N=240$

\begin{tabular}{|c|c|}
\hline Socio-demographic & $n(\%)$ \\
\hline \multicolumn{2}{|l|}{ Gender } \\
\hline Male & 59 (24.6) \\
\hline Female & $181(75.4)$ \\
\hline \multicolumn{2}{|l|}{ Ethnicity } \\
\hline Malay & $190(79.2)$ \\
\hline Chinese & $33(13.8)$ \\
\hline Indian & $15(6.3)$ \\
\hline Other & $2(0.8)$ \\
\hline \multicolumn{2}{|l|}{ Current residence } \\
\hline Hostel & $228(95.0)$ \\
\hline Home with family & $4(1.7)$ \\
\hline Rental & $8(3.3)$ \\
\hline \multicolumn{2}{|l|}{ Year of study } \\
\hline $1^{\text {st }}$ year & $83(34.6)$ \\
\hline $2^{\text {nd }}$ year & $76(31.7)$ \\
\hline $3^{\text {rd }}$ year & $81(33.8)$ \\
\hline \multicolumn{2}{|l|}{ Field of study } \\
\hline Arts & $58(24.2)$ \\
\hline Technical & $59(24.9)$ \\
\hline Sciences & $123(51.2)$ \\
\hline \multicolumn{2}{|l|}{ Faculty } \\
\hline Human Ecology (Art) & $58(24.2)$ \\
\hline Engineering (Technical) & $59(24.6)$ \\
\hline Biotechnology (Science) & $60(25.0)$ \\
\hline Medicine \& Health (Science) & $63(26.3)$ \\
\hline \multicolumn{2}{|l|}{ BMI category } \\
\hline Underweight $\left(<18.5 \mathrm{~kg} / \mathrm{m}^{2}\right)$ & 31 (12.9) \\
\hline Normal $(18.5-24.9$ kg/m²) & $147(61.3)$ \\
\hline Overweight $\left(\geq 25.0 \mathrm{~kg} / \mathrm{m}^{2}\right)$ & 47 (19.6) \\
\hline Obese $\left(\geq 30 \mathrm{~kg} / \mathrm{m}^{2}\right)$ & $15(6.3)$ \\
\hline
\end{tabular}

\section{Sleep quality according to PSQI}

Table 2 shows that 153 respondents $(63.8 \%)$ subjectively rated their overall sleep quality as fairly good, while only $1.3 \%$ rated it as very bad. Approximately two-third of the respondents reported very good $(33.3 \%)$ and fairly good $(35.0 \%)$ sleep latency, while the remaining onethird $(31.6 \%)$ reported taking more than 30 minutes to fall asleep. Only one-third 


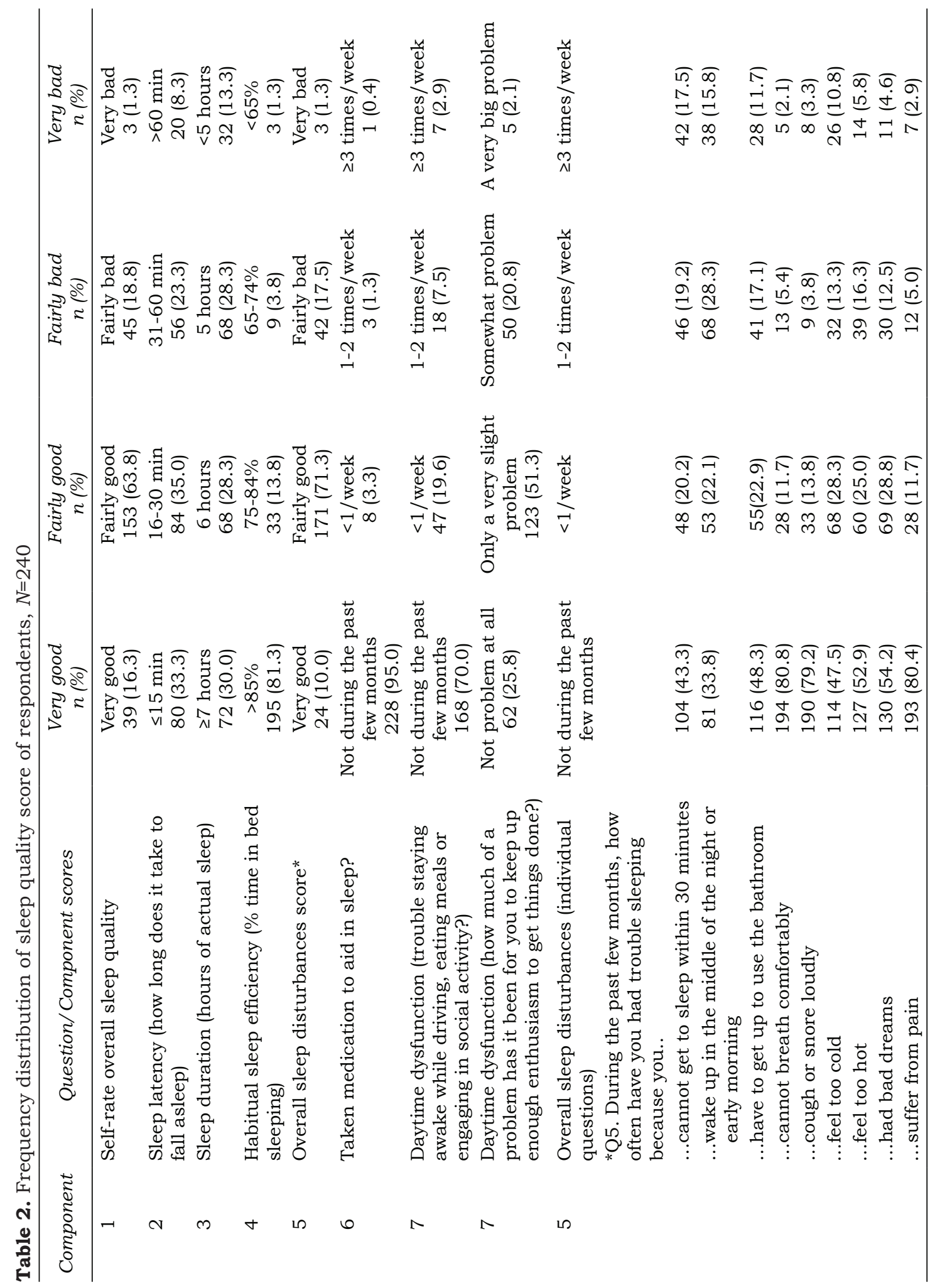


Table 3. Sleep quality of respondents according to body weight status, $N=240$

\begin{tabular}{lccccr}
\hline Sleep & $\begin{array}{c}\text { Underweight, } n=31 \\
\left(B M I<18.5 \mathrm{~kg} / \mathrm{m}^{2}\right)\end{array}$ & $\begin{array}{c}\text { Normal, } n=147 \\
\left(B M I 18.5-24.9 \mathrm{~kg} / \mathrm{m}^{2}\right)\end{array}$ & $\begin{array}{c}\text { Overweight, } n=47 \\
\left(B M I \geq 25 \mathrm{~kg} / \mathrm{m}^{2}\right)\end{array}$ & $\begin{array}{c}\text { Obese, } n=15 \\
\left(B M I \geq 30 \mathrm{~kg} / \mathrm{m}^{2}\right)\end{array}$ & $p$-value \\
quality & $n(\%)$ & $n(\%)$ & $n(\%)$ & $n(\%)$ & \\
\hline Good & $21(67.7)$ & $77(52.4)$ & $18(38.3)$ & $3(20.0)$ & $<0.001^{*}$ \\
Poor & $10(32.3)$ & $70(47.6)$ & $29(61.7)$ & $12(80.0)$ & \\
\hline
\end{tabular}

"Mann-Whitney U test significant at $p$-value $<0.05$

(30.0\%) of respondents experienced $\geq 7$ hours of adequate sleep duration, while $13.3 \%$ had very poor sleep duration (five hours or less per night) during the past month.

Majority of respondents $(81.3 \%)$ reported experiencing good to very good sleep, indicating very minimal sleep disturbances, while $18.8 \%$ of respondents experienced fairly bad to very bad sleep disturbances according to the PSQI algorithm. Based on specific responses to sleep disturbances, $44.1 \%$ of respondents reported waking up in the middle of the night or early morning at least once per week, $17.1 \%$ reported that they had bad dreams at least once per week. Among respondents who reported 'others' as their reason of sleep disturbances, at least once or twice per week was stress-related thoughts associated with anxiety, stress, overthinking, fatigue, and stressrelated symptoms including back pain, headache, and rapid heartbeats.

Meanwhile, $\quad 10.4 \%$ reported experiencing daytime dysfunction such as trouble staying awake while driving, eating meals or engaging in social activity at least once per week. More than half of the respondents (51.3\%) were slightly concerned about their day time dysfunction affecting their daily life, while $20.8 \%$ of respondents reported this as being somewhat a problem and five respondents $(2.1 \%)$ reported day time dysfunction heavily affected their daily life activity. Among the respondents, $1.7 \%$ reported having taken sleeping medication at least once per week within the past month.

\section{Association between components of sleep quality and body weight status}

Table 3 shows that there was a significant association between sleep quality and body weight status. More OW $(61.7 \%)$ and OB $(80.0 \%)$ respondents experienced poor sleep quality compared to those UW (32.3\%) and normal weight (NW) $(47.6 \%)$. The association between components of sleep quality and body weight status is shown in Table 4.

Results showed that Global PSQI, sleep latency, and sleep disturbances were associated with body weight status. Participants with OB had the highest score for Global PSQI (7.67 \pm 3.06$)$, sleep latency $(1.73 \pm 1.16)$, and sleep disturbances $(1.53 \pm 0.64)$ compared to those with other body weight statuses. Meanwhile, OW participants had a higher score for Global PSQI $(6.17 \pm 2.62)$ and sleep latency $(1.21 \pm 0.91)$ compared to UW (Global PSQI: 4.90 \pm 1.83 , sleep latency: $0.74 \pm 0.89$ ) and $\mathrm{NW}$ (Global PSQI:5.61 \pm 2.65 , sleep latency: $0.74 \pm 0.89)$ participants. This suggests that OW and OB are associated with poor sleep quality, longer sleep latency, and frequent experience of sleep disturbances compared to a leaner body weight status.

\section{DISCUSSION}

\section{Body weight status}

Almost a quarter of the respondents were classified in the body weight status of OW and OB. This result matched earlier studies reported in the Central region (Eow \& Gan, 2018; Nurismadiana \& Lee, 2018) and Northern region (Lai \& Say, 2013) of Peninsular Malaysia, but lower 
Table 4. Mean \pm SD scores of sleep quality and its component of PSQI according to body weight status $(N=240)$

\begin{tabular}{|c|c|c|c|c|c|}
\hline Sleep quality & $\begin{array}{c}\text { Underweight } \\
(n=31)\end{array}$ & $\begin{array}{l}\text { Normal } \\
(n=147)\end{array}$ & $\begin{array}{c}\text { Overweight } \\
(n=47)\end{array}$ & $\begin{array}{l}\text { Obese } \\
(n=15)\end{array}$ & p-value \\
\hline Global PSQI score & $4.90 \pm 1.83$ & $5.61 \pm 2.65$ & $6.17 \pm 2.62$ & $7.67 \pm 3.06$ & $0.01^{*}$ \\
\hline${ }^{\dagger} \mathrm{C} 1$ : Subjective sleep quality score & $0.84 \pm 0.69$ & $1.05 \pm 0.59$ & $1.19 \pm 0.71$ & $1.07 \pm 0.59$ & 0.12 \\
\hline${ }^{*} \mathrm{C} 2$ : Sleep latency score & $0.74 \pm 0.89$ & $1.02 \pm 0.92$ & $1.21 \pm 0.91$ & $1.73 \pm 1.16$ & $0.01^{*}$ \\
\hline C3: Sleep duration score & $1.19 \pm 1.05$ & $1.18 \pm 1.01$ & $1.34 \pm 1.03$ & $1.80 \pm 1.15$ & 0.17 \\
\hline C4: Habitual sleep efficiency score & $0.19 \pm 0.48$ & $0.22 \pm 0.51$ & $0.32 \pm 0.73$ & $0.40 \pm 0.91$ & 0.92 \\
\hline${ }^{\circ} \mathrm{C} 5$ : Sleep disturbances score & $0.94 \pm 0.36$ & $1.10 \pm 0.59$ & $1.09 \pm 0.50$ & $1.53 \pm 0.64$ & $0.01^{*}$ \\
\hline C6: Use of sleeping medication score & $0.06 \pm 0.25$ & $0.08 \pm 0.40$ & $0.04 \pm 0.20$ & $0.07 \pm 0.26$ & 0.97 \\
\hline C7: Daytime dysfunction score & $0.94 \pm 0.68$ & $0.97 \pm 0.67$ & $0.98 \pm 0.71$ & $1.07 \pm 0.59$ & 0.90 \\
\hline
\end{tabular}

Pittsburgh Sleep Quality Index (PSQI)

${ }^{\dagger} \mathrm{C} 1$-C7: Component scored from 0 to 3 , where higher score indicates poor sleep quality

"Kruskal-Wallis test significant at $p$-value $<0.05$

compared to that previously reported in the National Health and Morbidity Survey of young adults (age ranged between 20 to 24 years old) (IPH, 2015).

\section{Sleep quality}

Majority of university students in this study had a shorter sleeping duration than the recommended $\geq 7$ hours (Hirshkowitz et al., 2015). Mean global PSQI score suggested respondents from this study had better sleep quality than previously reported $(6.27 \pm 2.50$ hours $)$ in a study conducted among UPM and University of Malaya (UM) students (Tien Ngu et al., 2017). Tien Ngu et al. (2017) highlighted that lower sleep quality among the students of both universities could have been a result of the data collection being conducted during examination week. Meanwhile, this study collected data during the early semester after inter-semester break, presumably after the respondents have had adequate rest. However, regardless of the period of data collection, university students consistently have poor sleep quality.

Despite a poor mean score of sleep quality, the majority of respondents $(80.1 \%)$ in this study subjectively rated their overall sleep quality as 'good', based on their sleep quality in the past month. This suggested that majority of the respondents were not aware that they were experiencing poor sleep quality. This aligns with local (Tien Ngu et al., 2017) and western (Vargas, Flores \& Robles, 2014) studies that consistently showed university students were not aware of having poor sleep quality.

\section{Sleep quality and its components with body weight status}

This study suggested that there is a significant association between sleep quality with OW-OB. Results of the study are consistent with previous findings that supported the association between poor sleep quality and OW-OB (Krističevič, Štefan \& Sporiš, 2018; Peltzer \& Pengpid, 2017a; Fatima et al., 2016). Although causal links between poor sleep quality and OW-OB are still unclear, previous studies suggested that poor sleep quality has a substantial impact on psychology, leading to poor eating and healthrelated behaviours that subsequently promote the development of OW-OB. For instance, prolonged periods of being awake during night time increases the tendency of snacking (Spaeth, Dinges \& Goel, 2013). This behaviour is associated with a higher amount of calorie intake as most night time snacks contain fewer nutrients but are higher in energy density (Greer et al., 2013). Poor sleep quality 
also causes impairment in attention and poor judgement. With impaired decision making, individuals are more likely to make pleasure-seeking decisions and in this case, prefer foods with higher calorie densities (Ogilvie et al., 2017) or even impulsive eating in the absence of hunger (Greer et al., 2013). This further explains that to boost alertness, unrested and fatigue individuals tend to crave for snacks, consume caffeine and other sugar-sweetened beverages. Moreover, feeling tired during the day also impairs cognitive performance and lowers individual capacity to engage in physical activity (Štefan et al., 2018). Hence, poor sleep promotes poor eating behaviours accompanied by reduced energy expenditure, subsequently promoting the development of OW-OB.

The current study found that OW-OB respondents have significantly poorer sleep and experienced longer sleep latency (longer time was taken from full awareness to falling asleep) as compared to UW-NW respondents. These findings are in agreement with a study conducted among university students in Australia $(n=330$, mean age $27.42 \pm 10.36$ years) that found that OW-OB respondents had poorer sleep quality, characterised by shorter sleep duration and longer sleep latency (Yeh \& Brown, 2014). This has been further supported by a previous prospective cohort study that showed OW-OB respondents (aged between 21 to 35 years old) with higher body fat percentage experiencing poorer sleep quality marked by longer sleep latency, shorter sleep duration, and time to awaken compared to non-overweight and obese respondents (Wirth et al., 2015). However, the underlying mechanism behind such observation is yet to be known (Wirth et al., 2015).

OB students were found to experience more frequent sleep disturbances compared to their leaner counterparts. This result is in line with previous evidence suggesting that excessive weight was associated with a higher frequency of sleep disturbances including chocking, awakening, and unrested sleep (Vargas et al., 2014). Another study demonstrated that an elevation of six units in BMI increases the risk for obstructive sleep apnea (OSA), a condition that affects the continuity of sleep resulting in sleep disturbances, by four-times (QuintasNeves, Preto \& Drummond, 2016). Excessive weight especially visceral obesity affects metabolic hormones, influences secretion of inflammatory cytokines, promotes the severity of OSA that affects sleep-wake rhythm, and leads to sleep disturbances (Muscogiuri et al., 2016). Individuals with sleep disturbances have lowered sleep continuity, increased daytime fatigue, and favour an unhealthy lifestyle that further aggravates the development of obesity (Tubbs, Khader, Fernandez \& Grandner, 2020).

Several limitations should be taken into consideration when interpreting the findings of this study. The study design was cross-sectional, in which data collected among university samples was at a single point of time, therefore, the researcher was unable to draw conclusions on the cause and effect relationships between variables. Future research employing a longitudinal study design could better establish the temporal relationship between these variables. Qualitative studies could also be carried out to further explore the role of sleep quality in the development of obesity.

\section{CONCLUSION}

This research suggests that overweight and obese students at university are at greater risks of experiencing decreased sleep quality, having longer sleep latency and experiencing frequent sleep 
disturbances. Thus, there is a need for a wellness programme to reduce the incidence of obesity while promoting healthy sleeping habits among university students.

\section{Acknowledgements}

The authors are grateful to all respondents who participated in this study. This study has been funded by Universiti Putra Malaysia under the Putra Grant scheme (GP-IPS/2016/9514300).

\section{Authors' contributions}

NFS, conducted the study, data analysis and interpretation, prepared the draft of the manuscript; ZI, principle investigator, conceptualised and designed the study, supervised the project and reviewed the manuscript; SNAA \& SMN, supervised the project and reviewed the manuscript.

\section{Conflict of interest}

The authors have no conflict of interest.

\section{References}

Azad MC, Fraser K, Rumana N, Abdullah AF, Shahana N, Hanly PJ \& Turin TC (2015). Sleep disturbances among medical students: a global perspective. J Clin Sleep Med 11(1):69-74. https://doi.org/10.5664/jcsm.4370

Eow SY \& Gan WY (2018). Social media use, body image and body weight status: Comparison between university students with and without disordered eating in University Putra Malaysia. International Journal of Public Health and Clinical Sciences 5(1):129-145.

Fatima Y, Doi SAR \& Mamun AA (2016). Sleep quality and obesity in young subjects: a meta-analysis. Obes Rev 17(11): 1154-1166. https://doi.org/ 10.1111/obr.12444

Ganesh Kamath M, Prakash J, Dash S, Chowdhury S, Ahmed ZB \& Yusof MZZBM (2014). Is there an association between self-reported sleep duration, Body mass index and waist-hip ratio in young adults? A cross-sectional pilot study. $J$ Clin Diagnostic Res 8(9):5-7. https://doi. org/10.7860/JCDR/2014/8918.4808

Greer SM, Goldstein AN \& Walker MP (2013). The impact of sleep deprivation on food desire in the human brain. Nat Commun 4(2259): 1-7. https://doi.org/10.1038/ncomms3259
Hirshkowitz M, Whiton K, Albert SM, Alessi C, Bruni O, DonCarlos L, Hazen N, Herman J, Katz ES, Kheirandish-Gozal L, Neubauer DN, O`Donnell AE, Ohayon M, Peever J, Rawding R, Sachdeva RC, Setters B, Vitiello MV, Ware JC \& Adams Hillard PJ (2015). National sleep foundation's sleep time duration recommendations: Methodology and results summary. Sleep Health 1(1):40-43. https:// doi.org/10.1016/j.sleh.2014.12.010

IPH (2008). Nutritional Status. Report of the Third National Health and Morbidity Survey 2006 (NHMS III) (pp. 1-75). Institute for Public Health, Ministry of Health Malaysia, Kuala Lumpur.

IPH (2015). National Health and Morbidity Survey 2015 (NHMS 2015). Vol. II: Non-Communicable Diseases, Risk Factors \& Other Health Problems. Institute for Public Health, Ministry of Health Malaysia, Kuala Lumpur.

IPH (2019). National Health and Morbidity Survey (NHMS) 2019: Vol. I: NCDs - Non-Communicable Diseases: Risk Factors and other Health Problems. Institute for Public Health, Ministry of Health Malaysia, Kuala Lumpur.

Krističevič T, Štefan L \& Sporiš G (2018). The associations between sleep duration and sleep quality with body-mass index in a large sample of young adults. Int $J$ Environ Res Public Health 15(4). https://doi.org/10.3390/ ijerph 15040758

Kumar KA, Othman WA \& Jeppu AK (2020). Association of Epworth Sleepiness Score with Anthropometric Measurements in Malaysian University Students. Recent Advances in Biology and Medicine 6(2020):1-6.

Lai PP \& Say YH (2013). Associated factors of sleep quality and behavior among students of two tertiary institutions in Northern Malaysia. Med $J$ Malaysia 68(3):196-203.

Mohamad Nor NS, Ambak R \& Aris T (2018). An introduction to the My Body is Fit and Fabulous at home (MyBFF@home): A communitybased weight loss intervention study among Malaysian housewives. BMC Women's Health 18(Suppl 1):1-4. https://doi.org/10.1186/ s12905-018-0589-x

Musa NA, Moy FM \& Wong LP (2018). Prevalence and factors associated with poor sleep quality among secondary school teachers in a developing country. Ind Health 56(5):407-418. https:// doi.org/10.2486/indhealth.2018-0052 
Muscogiuri G, Barrea L, Annunziata G, Di Somma C, Laudisio D, Colao A \& Savastano S (2019). Obesity and sleep disturbance: the chicken or the egg? Crit Rev Food Sci Nutr, 59(13): 2158 2165. https://doi.org/10.1080/10408398.20 $\underline{18.1506979}$

Nurismadiana I \& Lee K (2018). Factors associated with sleep quality among undergraduate students at a Malaysian public university. International of Public Health and Clinical Sciences 5(6):373-391.

Ogilvie RP, Lutsey PL, Widome R, Laska MN, Larson N \& Neumark-Sztainer D (2017). Sleep indices and eating behaviours in young adults: Findings from Project EAT. Public Health Nutr 21(4):689-701. https://doi.org/10.1017/ S1368980017003536

Peltzer K \& Pengpid S (2017). Sleep duration, sleep quality, body mass index, and waist circumference among young adults from 24 low- and middle-income and two high-income countries. Int J Environ Res Public Health 14(6). https://doi.org/10.3390/ijerph14060566

Quintas-Neves M, Preto $J$ \& Drummond M (2016). Assessment of bariatric surgery efficacy on Obstructive Sleep Apnea (OSA). Rev Port Pneumol 22(6): 331-336. https://doi. org/10.1016/j.rppnen.2016.05.006.

Robinson HA, Dam R, Hassan L, Jenkins D, Buchan I \& Sperrin M (2019). Post-2000 growth trajectories in children aged $4-11$ years: A review and quantitative analysis. Prev Med Rep 14(March):1-14. https://doi.org/10.1016/j. pmedr.2019.100834

Spaeth AM, Dinges DF \& Goel N (2013). Effects of Experimental Sleep Restriction on Weight Gain, Caloric Intake, and Meal Timing in Healthy Adults. Sleep 36(7):981-990. https:// doi.org/10.5665/sleep. 2792

Štefan L, Sporiš G, Krističevič T \& Knjaz D (2018). Associations between sleep quality and its domains and insufficient physical activity in a large sample of Croatian young adults: a cross-sectional study. $B M J$ Open 8(7): e021902. https://doi.org/10.1136/ bmjopen-2018-021902
Tien Ngu S, Masalamany K, Abd Manan N \& Adam SK (2017). Sleep Quality among Pre-Clinical Medical Students in Universiti Putra Malaysia and Universiti Malaya, Malaysia. Education in Medicine Journal 9(3): 23-31. https://doi. org/10.21315/eimj2017.9.3.3

Tubbs AS, Khader W, Fernandez F, Grandner MA (2020). The common denominators of sleep, obesity, and psychopathology. Curr Opin Psychol 34: 84-88. https://doi.org/10.1016/j. copsyc.2019.11.003.

Vadeboncoeur C, Townsend N \& Foster C (2015). A meta-analysis of weight gain in first year university students: is freshman 15 a myth? BMC Obesity 2(22): 1-9. https://doi. org/10.1186/s40608-015-0051-7

Vargas PA, Flores M \& Robles E (2014). Sleep quality and body mass index in college students: The role of sleep disturbances. J Am Coll Health 62(8): 534-541. https://doi.org/ 10 .1080/07448481.2014.933344

Wirth MD, Hébert JR, Hand GA, Youngstedt SD, Hurley TG, Shook RP, Paluch AE, Sui X, James SL \& Blair SN (2015). Association between actigraphic sleep metrics and body composition. Ann Epidemiol 25(10): 773-778. https://doi. org/10.1016/j.annepidem.2015.05.001

WHO (2000). Obesity: Preventing and managing the global epidemic. In World Health Organization - Technical Report Series. https: / / doi.org/10.1016/S0140-6736(03)15268-3

Yeh SSS \& Brown RF (2014). Disordered eating partly mediates the relationship between poor sleep quality and high body mass index. Eat Behav 15(2):291-297. https://doi. org/10.1016/j.eatbeh.2014.03.014

Yunus RM, Wazid SW, Hairi NN, Choo WY, Hairi FM, Sooryanarayana R, Ahmad SN, Razak IA, Peramalah D, Aziz SA, Mohamad ZL, Mohamad R, Ali ZM \& Mahmud ABA (2017). Association between elder abuse and poor sleep: A cross-sectional study among rural older Malaysians. PLoS ONE 12(7):1-14. https:/ / doi. org/10.1371/journal.pone.0180222 